\title{
Friction Analysis for Piston Ring of Seal Device in the Stirling Engine
}

\author{
Hou Shunqiang ${ }^{1, a}$, Zhang Lili, ${ }^{2, b}$ and Zhang Xiaoyan ${ }^{3, c}$ \\ ${ }^{1}$ Qingdao Binhai University, Qingdao China \\ ${ }^{2}$ Shandong University of Science and Technology, Qingdao China \\ ${ }^{3}$ SAIC-GM-Wuling Automobile Co.Ltd SGMW , Liuzhou China \\ a hellospring111@163.com, ${ }^{b}$ sdkd_lili@126.com, ${ }^{c}$ xiaoyan1.zhang@sgmw.com.cn
}

Keywords: Stirling Piston ring; Dynamic seal; Friction and wear.

\begin{abstract}
The friction pair of piston ring-cylinder liner for the piston ring seal device of Stirling Engine is plastic-metal friction pair, its working conditions for dry friction. So the wear resistance of the piston ring is poor, its service life is short. This paper regard integral non-backpressure piston rings group as the research object in order to reduce wear: First, establishes auto-free lubricating mechanical model to piston rings group; Then, set up the mathematical model, which to meet the requirements of the sealing performance, for friction analysis; At last, Experimental analysis in the Piston Ring Seal Device of Stirling Engine to prove that this model is correct and available.
\end{abstract}

\section{Introduction}

The opening with back pressure free lubricating piston rings is used in most piston ring seal device of Stirling Engine in domestic at present. During the working process of piston rings with opening back pressure free lubricating, the compressed medium pressure will press on the surface of piston ring's inner column to make adhered pressure between piston ring and cylinder surface, which produce friction consumption in the process of piston rings' reciprocating movement. However, as the piston ring of Stirling Piston works in the dry friction condition, it is easy to wear and its service life is short, besides, the sealing performance of Stirling Engine is affected by the friction character directly. This paper mainly bases on the operation condition of Stirling Engine to analyze the elements that affect the wear of piston ring and propose some measures to lengthen piston ring's service life.

\section{Improved Sealing Structure of Stirling Engine Piston Ring}

The improved sealing structure of Stirling Engine piston ring is as Fig. 1. Two piston rings and two guide rings are designed on the piston. The piston ring has a slot and double loop with an inside and outside structure. Two pieces are included in the outside ring and one is the upper ring the anther one is the lower ring. The upper one is the open type one with straight incision structure. The lower one is an integrated ring with a convex at the corresponded point with upper incision. Those two rings form the ring group and make two ends adhere. The inside ring is a spring lamination made by a stainless steel belt piece and it is $10 \sim 15 \mathrm{~mm}$ longer than inside ring's perimeter. Curve it into a lap round to be a elastic ring when install it and then embed it into piston ring to form interference fit. The guide ring has a slot and a ring with an uninstall hole, which can help guide ring without gas pressure and only play the role of oriented support. The piston ring and guide ring are produced by the Chinese Academy of Sciences Lanzhou Institute of Chemical Physics and the compound design is made by $40 \% \mathrm{Cu}+60 \% \mathrm{~F} 4$ filled PTFE with self lubrication and good thermal conduction. With the force analysis as Fig. 2 and ignoring the affection that produced by surface roughness $\left(W_{A}=0\right)$ between piston ring and cylinder wall, the upper ring end adhered tightly with the lower ring end with influence of gas pressure. Both of the two rings have incision and convex to make these rings into a set without mutual turning. The inner perimeter and metal elastic ring of upper and lower rings has 
formed interference fit and the elastic ring's inner perimeter adheres tightly with piston ring's slot which also forms interference fit. The inner perimeters of upper and lower rings adhere tightly with metal-made elastic ring, which forms an integrated structure and make these two ends adhere tightly, and so does the elastic ring and metal ring's slot. In this way, the leakage path (1) and leakage path (2) will be filled it; because of the thermal expansion effect for the upper ring's opening, the upper ring's external diameter adheres with cylinder wall tightly and fill the leakage path (4). The straight incision on the upper ring forms to a clearance fit with the convex on the lower ring, which makes no mutual turning between these two rings. Due to the small clearance between the two ends, some gas that

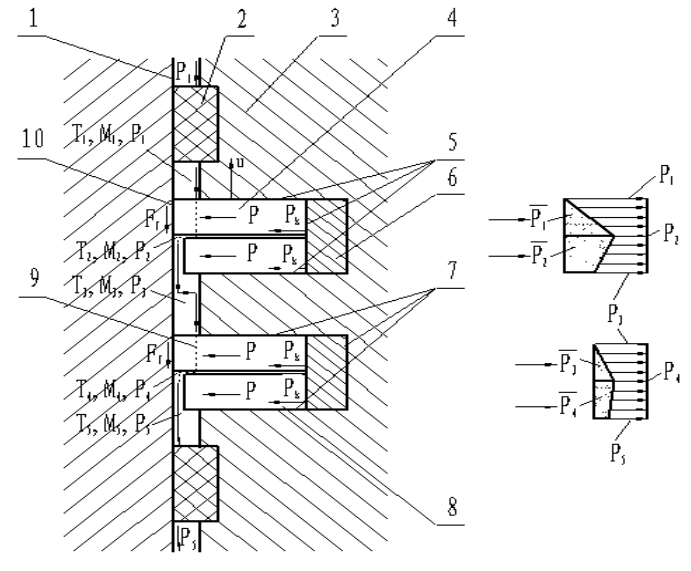
leaks from the clearance between upper ring's opening and lower ring's convex, leak into the clearance between lower ring and cylinder wall (gas cell 2and 4) to form the leakage path (3). The internal and external diameters of the guide ring form interference fit with the external of guide ring's slot and cylinder's internal diameter. However, the load-off hole is equipped on the guide ring and the guide ring does not have sealing function, the gas that goes through the clearance between guide ring and cylinder wall can not produce pressure drop, and the guide ring just play the role of guide.

1-cylinder liner 2- guide ring 3-piston 4-upper piston ring 5-leakage path(1) 6-elastic ring 7- leakage path(2) 8-lower piston ring 9- leakage path(3) 10- leakage path(4)

Fig.1 the structure diagram of no-back-pressure piston ring set

\section{Piston Rings' Friction Analysis}

The Elastic Specific Pressure Produces by Thermal Expansion.

The thermal expansion of piston can produce uniform pressure to the cylinder wall (Fig. 2)

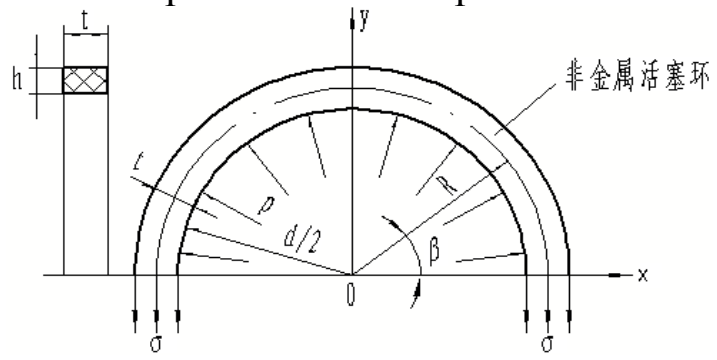

Fig. 2 Loaded diagram of no-back-pressure nonmetal piston

See the metal ring as rigid body when calculate thermal deformation. On the basis of Hooke's low during the working hours the tensile stress $\sigma$ of nonmetal ring is ${ }^{[4]}: \sigma=E \varepsilon=E\left(R-R_{0}\right) / R_{0}$

The loaded diagram of nonmetal ring during working hours is like drawing 4 . It can be known from mechanical equilibrium relation that the resultant force of tensile stress $\sigma$ on the y axis direction will equal to the component force that specific pressure $P$ work on the inside of nonmetal ring on the y axis. That is to say: $2 h t \sigma=\int_{0}^{\pi}\left(p h d_{j} \sin \beta / 2\right) d \beta=p h d_{j}$

Put the formula (1) into (2) to get the formula between the elastic specific pressure of piston rings, $\mathrm{P}$ and the working temperature $T_{1}: P=2 \operatorname{Et} \alpha\left(T_{1}-T_{0}\right) / d_{j}(i=1,2,3,4)$

The Effect from Gas Pressure to Piston Rings' Radial Direction Specific Pressure. 
The high pressure sealing gas through the leakage path (3) (two places) and other seal surfaces, its pressure reduces from $\mathrm{P} 1$ to $\mathrm{Pi}(\mathrm{i}=1,2,3,4)$ in sequence to corresponding gas cell; $\bar{P}_{m}$ is the average radial pressure that beard on the corresponding ring's external diameter's cylindrical surface and the pressure's direction points from external to internal, besides, as the effect of thermal expansion during working hours, the elastic ring produces outward radial specific pressure $P$ to the rings' internal diameter. Meanwhile, as the compression when first assembling, the guide ring also produces outward radial specific pressure $P_{\mathrm{k}}$ to cylinder walls. The algebraic sum of these pressures in radial direction is just the positive pressure that needed to form piston tings' static friction. The radial elastic specific pressure of elastic rings $P_{\mathrm{k}}$ can be calculated from formula $(4)^{[5]} \cdot P_{k}=\frac{E_{k} A_{e}}{7.08 D_{e}\left(D_{e} / t_{e}-1\right)}(4)$

The average radial pressure that beard by each external diameter's cylindrical surface will be seen as formula $(5)^{[6]} \cdot \bar{P}_{m}^{-}=\frac{1}{2}\left(P_{m}+P_{m+1}\right) \quad(m=1,2,3,4)$

The relation between average radial pressure and the sealed gas pressure $P_{1}$ will be seen as formula $(6)^{[6]}$.

$$
\bar{P}_{1}=0.76 P_{1}, \bar{P}_{2}=0.20 P_{1}, \bar{P}_{3}=0.076 P_{1}, \bar{P}_{4}=0.034 P_{1}
$$

The radial specific pressure from gas to piston rings is like the following: $P_{m}=\sum_{m=1}^{4} \bar{P}_{m}=1.07 P_{1}$

\section{The Effect from Pistons' Pace to Force of Sliding Friction.}

$F_{\mathrm{f}}$ means the static friction when piston rings in the station of static and sealed, it can be calculated from formula (8).

$$
F_{f}=\mu\left[\frac{8 E \cdot t \cdot \alpha\left(T_{1}-T_{0}\right)}{d_{j}}+\frac{E_{k} A_{e}}{1.77 D_{e}\left(D_{e} / t_{e}-1\right)}-1.07 P_{1}\right]=F_{f}\left(T_{1}, P_{1}\right)
$$

The piston rings' round surface and the cylinder walls form the relative sliding to produce sliding friction at sealed surface. We know that the size of sliding friction is related with the speed of relative movement of the things; therefore, the pace of pistons will affect the sliding friction (9)directly. When the system is sealed, the sliding friction can be expressed by the following function among working temperature $T_{1}$, sealing medium pressure $P_{1}$, piston pace $v$.

$$
F_{d}=F_{f}\left(T_{1}, P_{1}\right)+k_{1} v^{2}-k_{2} v=F_{d}\left(T_{1}, P_{1}, v\right)
$$

\section{Experimental Analyses}

The known physical quantity before experiment:

Chart 1 part of the certain experimental data

\begin{tabular}{|l|l|l|l|l|}
\hline$\mu=0.12$ & $E=280 \mathrm{MPa}$ & $\mathrm{t}=1.6 \mathrm{~mm}$ & $\alpha=(10 \sim 15) \times 10^{-5} \mathrm{~mm}$ & $\mathrm{~T}_{0}=25^{\circ} \mathrm{C}$ \\
\hline $\mathrm{dj}=55 \mathrm{~mm}$ & $\mathrm{E}_{\mathrm{k}}=193 \mathrm{MPa}$ & $\mathrm{A}_{\mathrm{e}}=0.2 \mathrm{~mm}$ & $\mathrm{D}_{\mathrm{e}}=51.8 \mathrm{~mm}$ & $\mathrm{t}_{\mathrm{e}}=1.75 \mathrm{~mm}$ \\
\hline $\mathrm{P}_{1}=0 \sim 8 \mathrm{MPa}$ & $\mathrm{K}_{1}=0.28$ & $\mathrm{~K}_{2}=247 \mathrm{~N} \cdot \mathrm{s} / \mathrm{m}$ & & \\
\hline
\end{tabular}

The mathematical computation model of friction and friction consumption power can be built according to the above formulas (1)-(9), and then find out the change regulation between the sliding friction $F_{\mathrm{d}}$ and other physical quantity. The measured dynamic friction in different operating conditions have been drawn curve graphs as the following Fig.3, Fig.4 and Fig.5.

\section{Conclusions}

(1)It can be seen from Fig. 3 that if the sealed gas pressure $P_{1}$ and piston pace v are constant, the force of sliding friction $F_{\mathrm{d}}$ will increase with the working temperature of piston rings and it will be 
steady at last. It is because when the working temperature increases the heat clearance of pistons' opening will enlarge and the external diameter of piston will adhere tightly with cylinder wall. Just right now, the radial positive pressure from piston ring to the cylinder wall increase and the static friction $F_{\mathrm{f}}$ aggrandizes. When the heat clearance of opening enlarges to limit, the static friction will to be maximum.

(2)It can be seen from Fig. 4 that if the sealed gas pressures $P_{1}$ and the working temperature $T_{1}$ are constant; when the pistons start to move its pace $v$ is very low, however, with the increase of $v$ the relative slip on the seal surface are severe and the radial wear quantity $h=k p v t$ of piston ring will increase, and the frictional vibration will be severe and the sliding friction $F_{\mathrm{d}}$ will be increased as well. When the radial wear quantity $h$ of piston ring to be the maximum, the contact surface between piston ring's external diameter and cylinder becomes very smooth and then the friction coefficient $\mu$ will decrease, the sliding friction $F_{\mathrm{d}}$ will decrease with decrease of static friction. When $\mu$ becomes minimum; $F_{\mathrm{f}}$ will be steady as well as sliding friction $F_{\mathrm{d}}$.

(3)It can be seen from Fig. 5 that if the piston pace $v$ and the working temperature $T_{1}$ are constant, the force of sliding friction $F_{\mathrm{d}}$ will decrease with the increasing of sealed gas pressure and then to be steady. It is because when $P_{1}$ increases the positive pressure $N$ will decrease and this can be reflected from formula (8). When the seal gas in cavity became thermal equilibrium state the seal gas pressure $P_{1}$ will to be steady. The force of static friction $F_{\mathrm{f}}$ will decrease to a steady state value and the sliding friction will be constant as well.

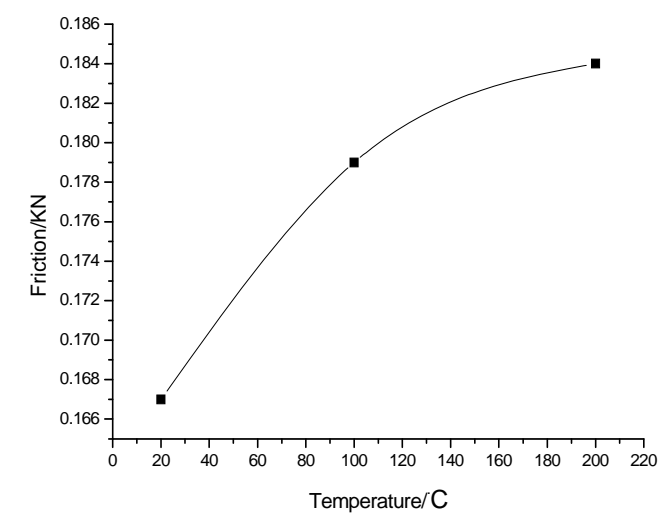

Fig. 3 curve between sliding friction $\mathrm{F}_{\mathrm{d}}$ and sealed working temperature $\mathrm{T}_{1}$ when $\mathrm{P}_{1}=8 \mathrm{MPa}, \nu=0.57 \mathrm{~m} / \mathrm{s}$

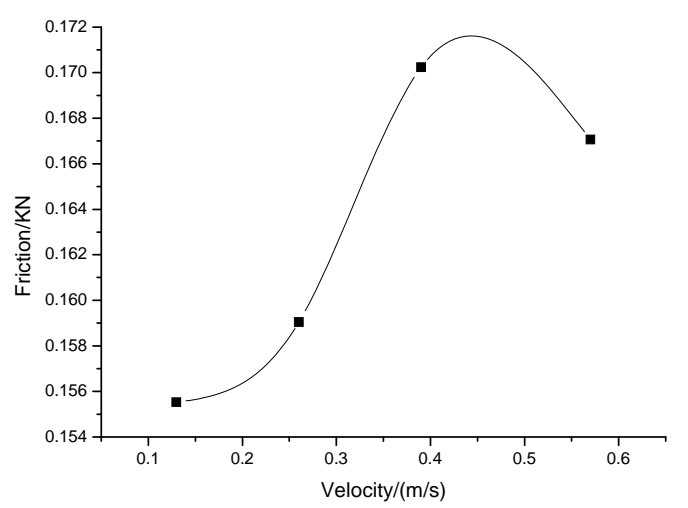

Fig. 4 curve between sliding friction $F_{\mathrm{d}}$ and piston pace $v$ when $T_{1}=150^{\circ} \mathrm{C}, P_{1}=2 \mathrm{MPa}$

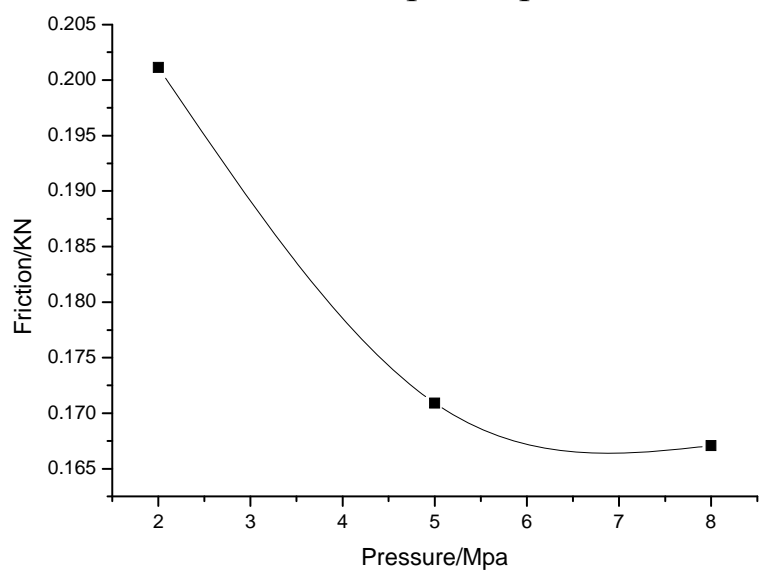

Fig. 5 curve between sliding friction $F_{\mathrm{d}}$ and sealed gas pressure $P_{1}$ whenv $=0.57 \mathrm{~m} / \mathrm{s}, T_{1}=150^{\circ} \mathrm{C}$

\section{References}

[1] Jin Donghan. Technology of Stiring Engine [M]. Harbin: Harbin Engineering University Press. 2009:162-166.

[2] Zhu Yufeng. Design \& Research of Entirety No-back-pressure Piston Ring on Compression 
Engine [J].Lubrication and Sealing. 2006, (12):106-107

[3] Li Yanlin. Calculation \& Practice of Free Lubrication Piston Rings' Sealing Parameter and Minimum Opening Clearance [J]. Sinkiang Oil Science and Technology, 1992, (3):84-87.

[4] Zhu Yufeng. Design \& Calculation of Working Clearance and Relative Interference Fit on No-back-pressure Piston Ring [N]. Hebei University of Science and Technology Journal, 2008-03-29(1):53-56.

[5] Peng Baocheng, Zhu Yufeng, etc. Affection Research of Elastic Rings to Piston Rings' Sealing and Service Life [J]. Lubrication and Sealing, 2006, (180): 97-98.

[6] Chen Geng, Jiao Guilong, Lu Dingji, etc. Tribology Design of Free Lubrication Compression Eengine Piston Ring [J].Shanghai Second Industrial University Journal, 1988,(1):1-8. 